\title{
Cocaine experience abolishes the motivation suppressing effect of CRF in the ventral midbrain
}

Idaira Oliva $^{1}$, Melissa M. Donate ${ }^{1}$, Merridee J. Lefner ${ }^{1}$, and Matthew J. Wanat ${ }^{1}$

${ }^{1}$ Neurosciences Institute and Department of Biology, University of Texas at San Antonio, San

Antonio, TX 78249, USA

\section{Corresponding Author:}

Matthew J. Wanat

Neurosciences Institute

Department of Biology

University of Texas at San Antonio

One UTSA Circle

San Antonio, TX 78249

Matthew.wanat@utsa.edu

210.458 .6684 


\begin{abstract}
:
Stress affects dopamine-dependent behaviors in part through the actions of corticotropin releasing factor (CRF) in the ventral tegmental area (VTA). For example, acute stress engages CRF signaling in the VTA to suppress the motivation to work for food rewards and promote drug seeking behavior. These diverging behavioral effects in food- and drug-based tasks could indicate that CRF modulates goal-directed actions in a reinforcer-specific manner. Alternatively, prior drug experience could functionally alter how CRF in the VTA regulates dopaminedependent behavior. To address these possibilities, we examined how intra-VTA injections of CRF influenced cocaine intake and whether prior drug experience alters how CRF modulates the motivation for food rewards. Our results demonstrate that intra-VTA injections of CRF had no effect on drug intake when self-administering cocaine under a progressive ratio reinforcement schedule. We also found that a prior history of either contingent or non-contingent cocaine infusions abolished the capacity for CRF to reduce the motivation for food rewards. Furthermore, voltammetry recordings in the nucleus accumbens illustrate that CRF in the VTA had no effect on cocaine-evoked dopamine release. These results collectively illustrate that exposure to abused substances functionally alters how neuropeptides act within the VTA to influence motivated behavior.
\end{abstract}




\section{Introduction:}

Stress induces the release of corticotropin releasing factor (CRF), which is responsible for initiating the hormonal and physiological responses to stress [1]. CRF signaling within the mesocorticolimbic system also mediates the effects of stress on motivation and decision-making processes [2]. For example, CRF receptor activation within the ventral tegmental area (VTA) is required for the stress-induced reduction in the motivation to work for food rewards [3]. Acute stress also decreases the preference for high effort / large reward options in decision making tasks [4]. This alteration in effort-based decision making is blocked by antagonizing CRF receptors and recapitulated by administering CRF directly into the VTA [4]. Therefore, the actions of CRF in the ventral midbrain are critical for how stress regulates behavioral responding for natural rewards.

Whereas acute stress reduces the motivation to work for food rewards, stress promotes the reinstatement of drug seeking in a CRF-dependent manner [5]. Antagonizing CRF receptors in the VTA prevents stress-induced cocaine seeking [6,7]. Furthermore, intra-VTA injections of CRF are sufficient to reinstate cocaine seeking in the absence of stress [6,7], which together highlights the involvement of midbrain CRF signaling in how stress promotes drug-dependent behaviors.

How does CRF act within the VTA to suppress the motivation for food rewards and also enhance drug seeking behavior? One possibility is that CRF influences behavior in a reinforcerspecific manner. Since abused substances alter the electrophysiological effects of CRF on dopamine neurons [8-11], an alternative possibility is that prior drug experience changes how CRF in the VTA regulates dopamine-dependent behavior. Here, we performed experiments to determine whether the behavioral effects of CRF in the VTA are reinforcer-specific or regulated by prior drug intake. Rats were either (i) trained to nosepoke for cocaine infusions, (ii) received yoked cocaine infusions, or (iii) were drug naïve before they were trained to lever press for food rewards. We examined how intra-VTA CRF injections affected the motivation to work for cocaine infusions and food rewards under a progressive ratio (PR) reinforcement schedule. In this manner, we could determine if CRF differentially influenced the motivation to work for drug and food rewards within the same subjects. Additionally, we could ascertain if a prior history of 
contingent or non-contingent drug experience altered how CRF regulated the motivation for food rewards.

\section{Methods:}

\section{Subjects and surgery}

All procedures were approved by the Institutional Animal Care and Use Committee at the University of Texas at San Antonio. Male Sprague-Dawley rats (Charles River, MA) were pairhoused upon arrival and given ad libitum access to water and chow and maintained on a 12-hour light/dark cycle. Surgeries were performed under isoflurane anesthesia on rats weighing between 300-350 g. For intracranial surgeries, rats were implanted with a bilateral guide cannula targeting the VTA (relative to bregma: $5.6 \mathrm{~mm}$ posterior; $\pm 0.5 \mathrm{~mm}$ lateral; $7.0 \mathrm{~mm}$ ventral). Rats used for voltammetry recordings were additionally implanted with carbon fiber electrodes in the nucleus accumbens (relative to bregma: $1.3 \mathrm{~mm}$ anterior; $\pm 1.3 \mathrm{~mm}$ lateral; $7.0 \mathrm{~mm}$ ventral) along with a $\mathrm{Ag} / \mathrm{AgCl}$ reference electrode placed at a convenient location. All components were secured in place with cranioplastic cement. Rats were single housed following the intracranial implantation surgery and for the duration of the experiment. Intravenous jugular catheter surgeries were performed 1-3 weeks following the intracranial surgery. Rats assigned to the yoked cue control group underwent a mock catheter surgery in which they only received a backmount cannula implant. All animals were allowed to recover for at least 1 week following the catheter surgery before initiating training.

\section{Cocaine self-administration training}

Cocaine self-administration sessions were performed in operant boxes (Med Associates) with grid floors, a houselight, two nosepoke ports with lights, and a tone generator, as described previously [12]. Behavioral sessions (1 hr) began with the illumination of the houselight and were performed only once per day. After completing the required number of nosepokes into the active port, rats received a $0.3 \mathrm{mg} / \mathrm{kg}$ i.v. infusion of cocaine, the tone and active nosepoke light turned on $(5 \mathrm{~s})$, which coincided with the initiation of a $20 \mathrm{~s}$ timeout period (houselight off). Nosepokes during the timeout or into the inactive port had no consequences. Rats were first 
trained to self-administer cocaine on a fixed ratio - 1 (FR1) reinforcement schedule. After rats completed at least $10 \mathrm{FR} 1$ sessions and earned $\geq 10$ infusions per day for at least two consecutive sessions, they were then trained to self-administer cocaine under a PR reinforcement schedule in which the operant requirement escalated according to the following equation: operant requirement $=5 * \mathrm{e}^{\text {(infusion number } * 0.2)}-5$. Intra-VTA microinjections were performed once rats achieved $<15 \%$ variance in the infusions earned over three consecutive sessions. Rats failing to earn at least 6 infusions during PR sessions were excluded from the study. Yoked cocaine rats underwent 15 training sessions in which they received 23 infusions, which was based upon the performance of a rat that had self-administered cocaine. The yoked cocaine delivery was accompanied by the tone and active nosepoke light turning on $(5 \mathrm{~s})$ and the houselight turning off for $20 \mathrm{~s}$, which was identical to the conditions in rats that self-administered cocaine. Yoked cue rats underwent the identical training procedures as the yoked cocaine rats (including backmount attachment to the infusion tubing), though they did not receive cocaine infusions.

\section{Food lever press training}

Lever press training for food rewards was performed after cocaine self-administration experiments, 15 sessions of non-contingent cocaine infusions (yoked cocaine), or 15 sessions of non-contingent cue presentations (yoked cue). Rats were placed and maintained on mild food restriction ( $\sim 15 \mathrm{~g}$ /day of standard lab chow) to target $90 \%$ free-feeding weight, allowing for an increase in weight of $1.5 \%$ per week. Experimental 45-mg food pellets (F0021, BioServ, NJ) were placed in their home cages on the day prior to the first training session to familiarize the rats with the food pellets. Behavioral sessions were performed in operant chambers that had grid floors, a house light, and contained a food tray and two cue lights above two retractable levers on a single wall. The cue lights and their corresponding levers were located on either side of the food tray. Rats were first trained to lever press for food rewards during sessions in which a single lever was presented throughout the duration of the session and a single lever press resulted in the delivery of the food reward. After rats successfully completed this training session (100 pellets earned within 90 mins), they were then trained on sessions in which both levers were extended and the cue light was illuminated over the active lever. Completion of the correct number of 
lever presses led to the delivery of the food reward, retraction of the levers, the cue and house lights turning off and a light over the food tray turning on for a $30 \mathrm{~s}$ inter-trial interval. Behavioral sessions consisted of a total of 60 trials and were performed only once per day. Rats were first trained to complete 60 trials on an FR1, FR2 and FR4 reinforcement schedule before progressing to a PR reinforcement schedule. PR sessions were identical to FR sessions except that the operant requirement on each trail $(\mathrm{T})$ was the integer (rounded down) of 1.4(T-1) lever presses starting at 1 lever press, as described previously [3,13]. Food PR sessions ended after 15 min elapsed without completion of the response requirement in a trial. Note that lever press responding is calculated as a rate to normalize behavioral performance across animals that differ in the duration of the food PR session. Intra-VTA microinjections were performed once rats achieved $<15 \%$ variance in the food pellets earned over three consecutive sessions.

\section{Voltammetry recordings}

Chronically-implanted carbon-fiber microelectrodes were connected to a head-mounted voltammetric amplifier for dopamine detection in behaving rats using fast-scan cyclic voltammetry as described previously $[3,12,14]$. The potential applied to the carbon fiber was ramped in a triangle wave from $-0.4 \mathrm{~V}(\mathrm{vs} \mathrm{Ag} / \mathrm{AgCl})$ to $+1.3 \mathrm{~V}$ and back at a rate of $400 \mathrm{~V} / \mathrm{s}$ during a voltammetric scan and held at $-0.4 \mathrm{~V}$ between scans at a frequency of $10 \mathrm{~Hz}$. Dopamine was isolated from the voltammetry signal using chemometric analysis [15], using a standard training set accounting for dopamine, $\mathrm{pH}$, and background drift. The dopamine concentration was estimated based on the average post-implantation sensitivity of electrodes (34 nA/ $\mu \mathrm{M})$ [14]. Peak cocaine-evoked dopamine release was calculated for the $100 \mathrm{~s}$ following a single $1.8 \mathrm{mg} / \mathrm{kg}$ i.v. cocaine infusion [16].

\section{Intra-VTA microinjections and data analysis}

Rats received bilateral $0.5 \mu \mathrm{l}$ injections of CRF (Bachem) or ACSF (Tocris) into the VTA at a rate of $250 \mathrm{nl} / \mathrm{min}$. The injector was removed from the brain after at least $1 \mathrm{~min}$ had elapsed since the end of the infusion. Rats were placed in their homecage for $20 \mathrm{~min}$ before starting behavioral sessions. The dose-dependent effect of CRF injections on behavior were assessed in a 
counterbalanced manner. Intra-VTA injections were separated by at least one session in which no injections were performed. Additionally, subsequent injections were not performed until the rewards earned during the behavior-only PR sessions came within $15 \%$ of the average rewards earned during the three baseline sessions. Statistical analyses utilized a one-way ANOVA followed by post-hoc Dunnett's test relative to the ACSF injection. The Geisser-Greenhouse correction was applied to address unequal variances between the treatments for repeated measures ANOVAs.

\section{Histology}

Histology was performed to verify the placement of guide cannula and voltammetry electrodes (Supplemental Fig. 1). Rats were intracardially perfused with 4\% paraformaldehyde and brains were removed and post-fixed in the paraformaldehyde solution for at least $24 \mathrm{~h}$. Brains were subsequently placed in $15 \%$ and 30\% sucrose solutions in phosphate-buffered saline. Brains were then flash frozen in dry ice, coronally sectioned and stained with cresyl violet.

\section{Results:}

Male rats were trained to nosepoke for cocaine infusions and then to lever press for food rewards. In this manner we could ascertain how CRF acts within the VTA to influence the motivation to work for drug and food rewards in the same animals (Fig. 1A). Rats selfadministered cocaine $(0.3 \mathrm{mg} / \mathrm{kg}$ i.v. $)$ on an FR1 reinforcement schedule (mean $11.2 \pm 0.7$ training sessions, $\mathrm{n}=10$ rats) before self-administering cocaine on a PR reinforcement schedule (mean $10.1 \pm 1.1$ training sessions). Animals then received bilateral intra-VTA injections of CRF $(0-1 \mu \mathrm{g})$ prior to cocaine PR sessions. Local injections of CRF into the VTA had no effect on the number of cocaine infusions earned (one-way repeated measures ANOVA $\mathrm{F}_{(2.3,20.4)}=0.2, \mathrm{p}=$ 0.83; Fig. 1B), the number of active nosepokes (one-way repeated measures $\operatorname{ANOVA~F}_{(2.1,18.8)}=$ $0.1, \mathrm{p}=0.88$; Fig. $1 \mathrm{C}$ ), or the number of inactive nosepokes during cocaine PR sessions (oneway ANOVA $F_{(1.4,19.9)}=0.3, p=0.70$; Fig. 1D). Rats were then trained to lever press for food rewards, before assessing how intra-VTA CRF injections $(0-1 \mu \mathrm{g})$ affected the motivation to 
B

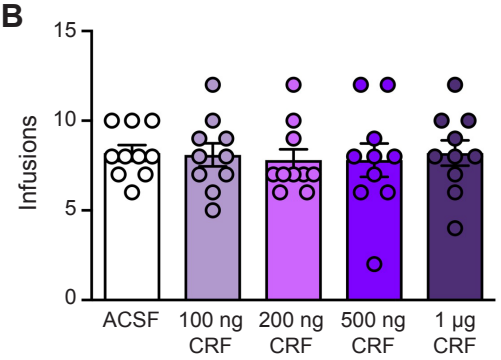

E

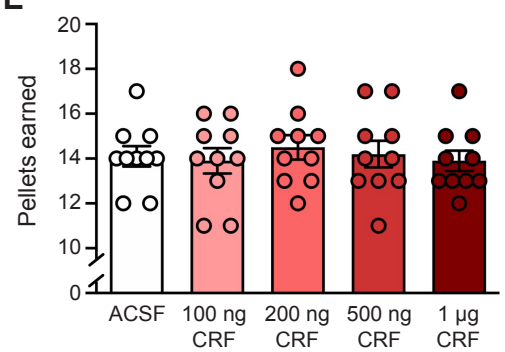

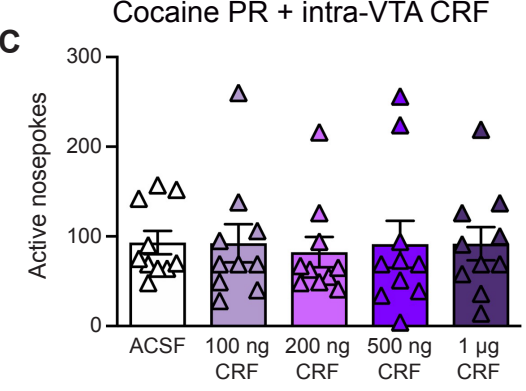

Food PR + intra-VTA CRF

$\mathbf{F}$

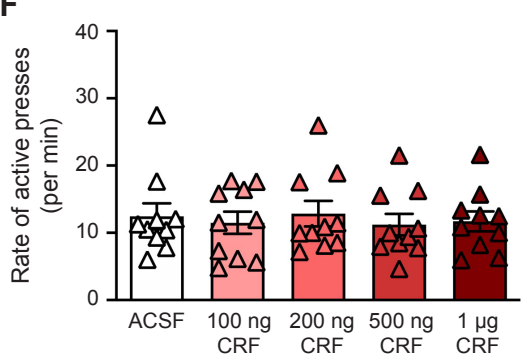

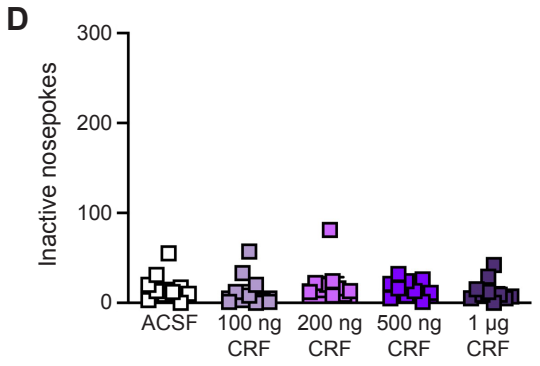

G

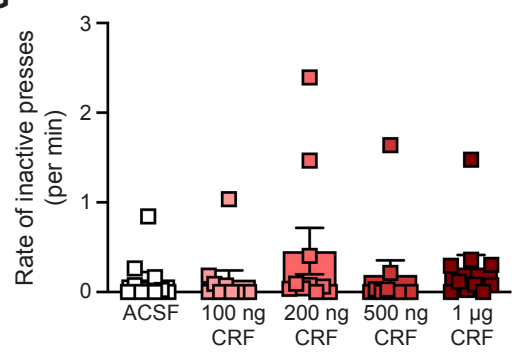

Figure 1. CRF does not affect the motivation for drug or food rewards following cocaine selfadministration. (A) Outline of training for rats self-administered cocaine. (B-D) Effect of intra-VTA injections of CRF on cocaine infusions (B), active nosepokes (C), and inactive nosepokes (D) during cocaine self-administration sessions under a PR reinforcement schedule. (E-G) Effect of intra-VTA injections of CRF on food pellets earned (E), rate of active lever presses $(F)$, and rate of inactive lever presses $(\mathrm{G})$ during operant responding for food rewards under a PR reinforcement schedule.

work for food rewards on a PR reinforcement schedule (Fig. 1A). Our results demonstrate that CRF did not influence the number of food rewards earned (one-way repeated measures ANOVA $\mathrm{F}_{(2.0,17.8)}=0.6, \mathrm{p}=0.55$; Fig. 1E), the rate of active lever presses (one-way repeated measures $\operatorname{ANOVA~}_{(2.4,21.9)}=0.4, \mathrm{p}=0.71$; Fig. $1 \mathrm{~F}$ ), or the rate of inactive lever presses (one-way repeated measures ANOVA $\mathrm{F}_{(2.1,18.7)}=1.1, \mathrm{p}=0.37$; Fig. 1G). Although CRF acts within the VTA to inhibit the motivation to work for food rewards in drug-naïve animals [3], we find that CRF does not affect the motivation to work for cocaine infusions or for food rewards in animals with prior drug experience.

The inability for CRF to regulate the motivation for food rewards following a history of cocaine self-administration could be due to the pharmacological actions of cocaine or alternatively could require contingent drug intake. To address these possibilities, a separate cohort of rats received yoked cocaine infusions along with the corresponding cocaine delivery 

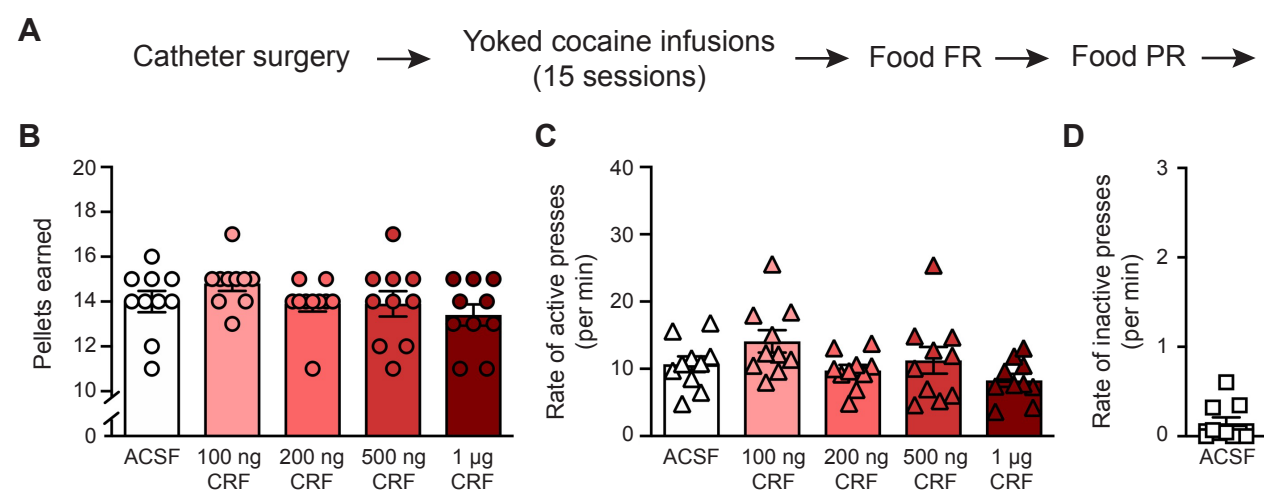

Food PR +

intra-VTA CRF

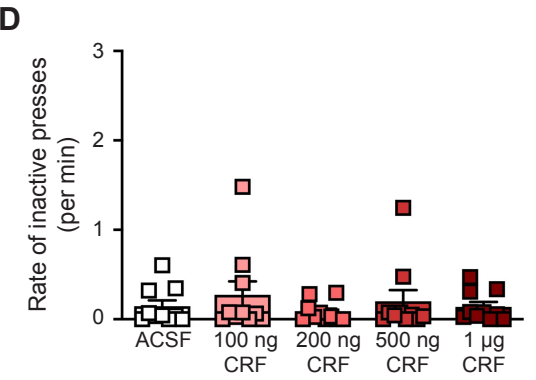

Figure 2. CRF does not affect the motivation for food rewards following non-contingent cocaine infusions. (A) Outline of training for yoked cocaine treated rats. (B-D) Effect of intra-VTA injections of $\mathrm{CRF}$ on food pellets earned (B), the rate of active lever presses (C), and the rate of inactive lever presses (D) during operant responding for food rewards under a PR reinforcement schedule in rats that had received yoked cocaine infusions.

cues $(\mathrm{n}=10$ rats). Following 15 sessions of non-contingent cocaine infusions, rats were trained to lever press for food pellets before we assessed how intra-VTA CRF injections influenced the motivation to work for food rewards (Fig. 2A). Identical to the results from the cocaine selfadministering rats (Fig. 1), CRF injections had no effect on the number of food pellets earned (one-way repeated measures ANOVA $\mathrm{F}_{(2.1,19.1)}=2.0, \mathrm{p}=0.16$; Fig. $2 \mathrm{~B}$ ), the rate of active lever presses (one-way repeated measures ANOVA $\mathrm{F}_{(2.8,25.4)}=3.0, \mathrm{p}=0.05$; Fig. $2 \mathrm{C}$ ), or the rate of inactive lever presses during food PR sessions in rats that had received yoked cocaine infusions (one-way repeated measures ANOVA $\mathrm{F}_{(1.9,17.0)}=0.6, \mathrm{p}=0.58$; Fig. 2D). Therefore, the pharmacological effects of cocaine are sufficient to prevent the avolitional influence of CRF in the VTA.

We next sought to exclude the possibility that the catheter surgery and/or exposure to the cues contributed to the change in the capacity for CRF to regulate motivation. To address this, a third group of rats underwent a mock catheter surgery followed by sessions in which they received the yoked presentation of cues ( $n=12$ rats). The yoked cue presentations were identical to the cues used from the self-administration / yoked cocaine experiments, except that no drug was delivered. Following 15 yoked cue presentation sessions, rats were trained to lever press for food rewards before assessing how intra-VTA CRF injections influenced the motivation to work for food (Fig. 3A). We found that CRF dose-dependently reduced the number of food pellets earned (one-way repeated measures $\operatorname{ANOVA~}_{(2.5,27.0)}=13.0, \mathrm{p}<0.0001$; post-hoc Dunnett's test 

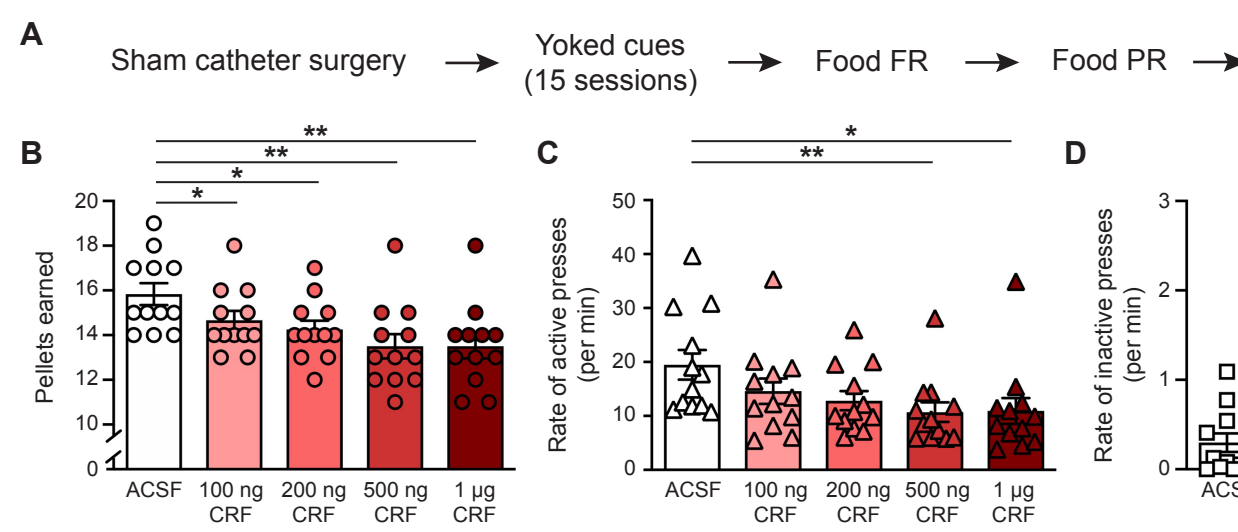

Figure 3. CRF inhibits the motivation to work for food rewards in drug-naïve animals. (A) (A) Outline of training for yoked cue rats. (B-D) Effect of intra-VTA injections of CRF on food pellets earned (B), rate of active lever presses (C), and rate of inactive lever presses (D) during operant responding for food rewards under a PR reinforcement schedule in rats that had received yoked presentations of cues. $* p<0.05, * * p<0.01, * * * p<0.001$.

relative to ACSF: $100 \mathrm{ng}, \mathrm{q}_{11}=3.0, \mathrm{p}<0.05 ; 200 \mathrm{ng}, \mathrm{q}_{11}=3.5, \mathrm{p}<0.05 ; 500 \mathrm{ng} \mathrm{q}_{11}=4.6, \mathrm{p}<$ $0.01 ; 1 \mu \mathrm{g} \mathrm{q} 11=4.4, \mathrm{p}<0.01$; Fig. 3B). The suppression in rewards earned was accompanied by a dose-dependent reduction in the rate of active lever presses (one-way repeated measures $\operatorname{ANOVA~F}_{(2.2,23.9)}=7.8, \mathrm{p}=0.0019$; post-hoc Dunnett's test relative to ACSF: $100 \mathrm{ng}, \mathrm{q}_{11}=2.4, \mathrm{p}$ $>0.05 ; 200 \mathrm{ng}, \mathrm{q}_{11}=2.5, \mathrm{p}>0.05 ; 500 \mathrm{ng} \mathrm{q} 11=4.1, \mathrm{p}<0.01 ; 1 \mu \mathrm{g} \mathrm{q} 11=3.4, \mathrm{p}<0.05 ;$ Fig. 3C). There was a main effect of intra-VTA CRF injections on the rate of inactive lever presses, though post-hoc analyses found no difference between ACSF injections and CRF injections at any of the tested doses (one-way repeated measures ANOVA $\mathrm{F}_{(1.8,20.0)}=3.9, \mathrm{p}=0.04$; Fig. 3D).

The inability for CRF to affect the motivation for food rewards in cocaine-treated rats was not due to intrinsic differences in behavioral responding between the groups. Specifically, there was no difference between the yoked cue, yoked cocaine, and cocaine self-administration groups in the rewards earned (one-way ANOVA $\mathrm{F}_{(2,29)}=1.0, \mathrm{p}=0.38$; Fig. 4A), the rate of active presses (one-way ANOVA $\mathrm{F}_{(2,29)}=1.0, \mathrm{p}=0.40$; Fig. 4B), or the rate of inactive lever presses (one-way ANOVA $\mathrm{F}_{(2,29)}=0.2, \mathrm{p}=0.81$; Fig. 4C) during the baseline PR sessions prior to intraVTA CRF injections. Together, these data demonstrate that CRF acts within the midbrain to inhibit the motivation to work for food rewards only in drug-naïve animals.

Dopamine transmission in the nucleus accumbens is required for engaging in high-effort behaviors [17]. Conversely, suppressing dopamine neuron activity at the time of the reward delivery reduces the motivation to work for rewards [18]. In drug naïve animals, CRF acts within 

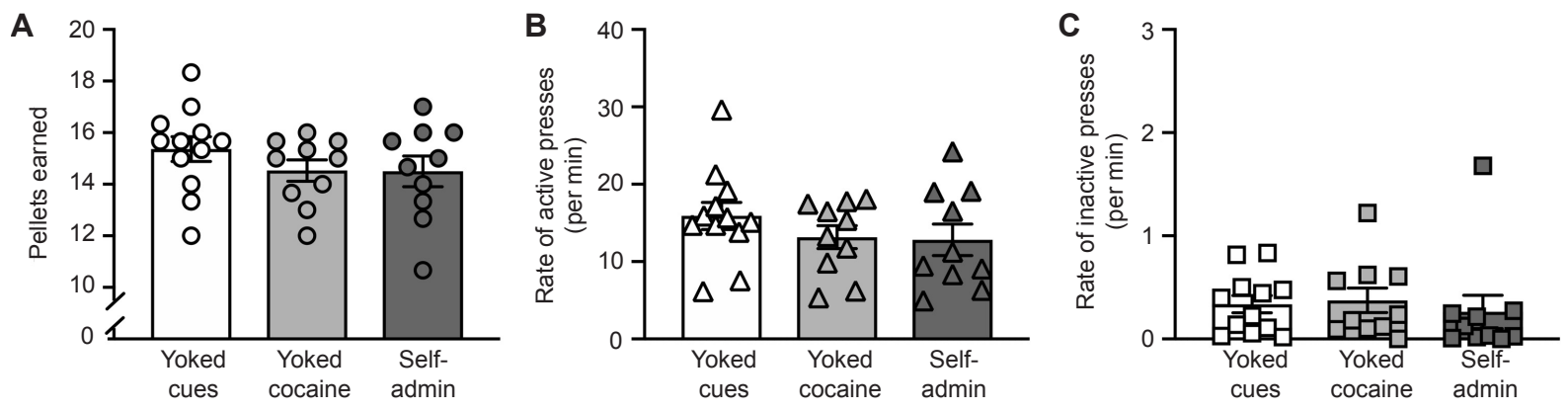

Figure 4. No behavioral differences between groups during baseline food PR sessions. (A-C) The relative behavioral performance between the cocaine self-administration, yoked cocaine, and yoked cue rats during baseline PR sessions. There was no difference in food pellets earned (A), the rate of active lever presses (B), and rate of inactive lever presses (C).

the VTA to attenuate dopamine release to food rewards [3]. Since midbrain CRF does not influence motivation following exposure to cocaine, we anticipated that CRF would not affect dopamine release to drug rewards in cocaine experienced animals. To test this prediction, we performed voltammetry recordings in the nucleus accumbens of dopamine release to an infusion of cocaine $(1.8 \mathrm{mg} / \mathrm{kg}$ i.v.). Rats were habituated to this procedure (minimum 10 prior cocaine infusions) before assessing how intra-VTA injections of CRF influenced cocaine-evoked dopamine release. Although dopamine release to drug rewards is controlled by neuronal activity within the VTA $[16,19]$, CRF in the VTA had no effect on dopamine release to cocaine infusions (oneway $\operatorname{ANOVA~}_{(4,22)}=0.4, \mathrm{p}=0.84$; $\mathrm{n}=6$ electrodes, Fig. 5A-C). These findings along with our prior work [3], illustrates the capacity for midbrain CRF to regulate rewardevoked dopamine release is related to
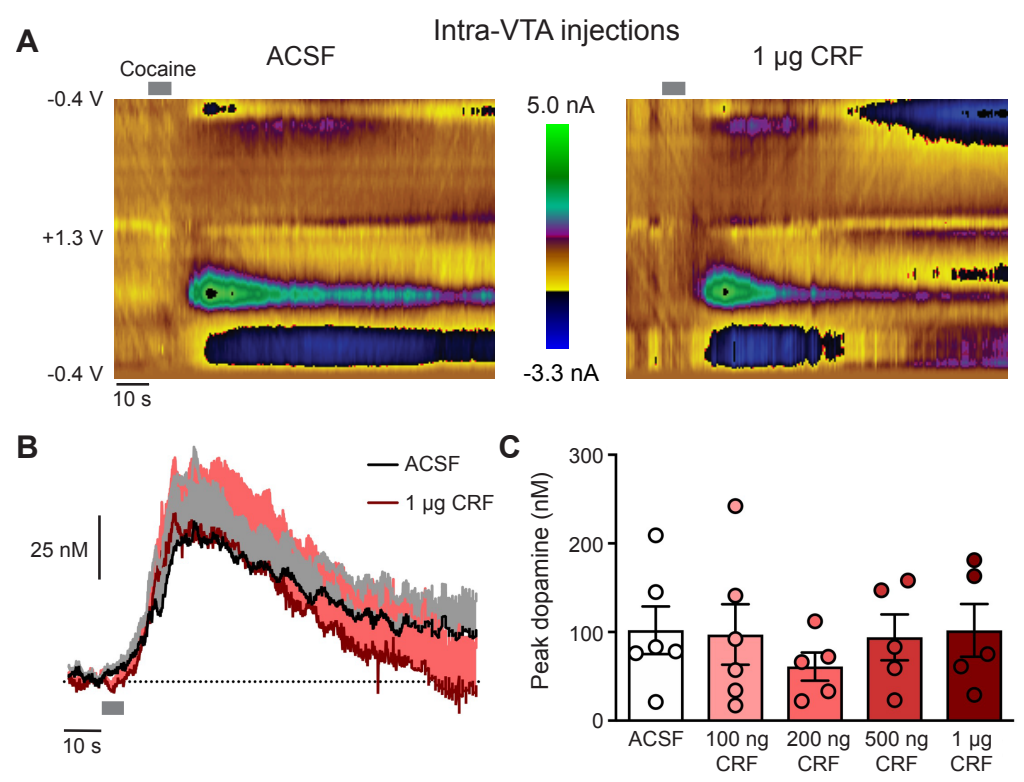

Figure 5. Intra-VTA injections of CRF do not affect cocaine-evoked dopamine release. (A) Representative colorplots of voltammetry recordings from a single electrode of cocaine-evoked dopamine release after intra-VTA injections of ACSF (left) or $1 \mu \mathrm{g}$ CRF (right). (B) Average dopamine response to the cocaine infusion across electrodes (C) Peak dopamine release to the cocaine infusion. 
its ability to influence motivated behavior.

\section{Discussion:}

Acute stress influences a host of behaviors through the actions of CRF in the VTA, with effects on motivation, decision-making, subsequent drug intake, and drug seeking [3,4,6,7,20-22]. Intriguingly, midbrain CRF suppresses dopamine-dependent behaviors in drugnaïve animals $[3,4]$, whereas CRF promotes dopamine-dependent behaviors in drug-experienced animals [6,7,20-22]. These conflicting reports indicate that the behavioral effects of CRF in the midbrain are either reinforcer-specific, or alternatively depend upon prior drug experience. Our results support the latter, as we demonstrate that the capacity for midbrain CRF to reduce the motivation for food rewards is lost following a history of cocaine self-administration or noncontingent cocaine infusions. Although CRF promotes the reinstatement of drug seeking in rats that have undergone extinction training [6,7], we find that midbrain CRF does not affect the motivation to work for cocaine during self-administration sessions. Therefore, the functional impact of CRF in the VTA on drug-dependent behaviors may depend upon if the reinforcer can be earned.

The motivation to work for rewards is enhanced by augmenting mesolimbic dopamine transmission and attenuated by inhibiting dopamine signaling [23-26]. In particular, motivation in appetitive tasks is influenced by dopamine transmission at the time of the reward delivery [18]. Large rewards evoke greater dopamine release relative to small rewards, which parallels the greater effort rats will exert to obtain larger rewards [3]. Conversely, the CRF-mediated reduction in motivation to work for food rewards is accompanied by a decrease in reward-evoked dopamine release [3]. We found that intra-VTA injections of CRF do not affect the motivation to work for cocaine infusions or dopamine release to cocaine infusions. Together, these findings demonstrate that the motivation to work for a given reward is intimately linked to how the dopamine system responds to the reward delivery.

Although midbrain CRF attenuates reward-evoked dopamine release in drug-naïve animals [3], it is likely that CRF is not solely acting upon dopamine neurons within the VTA. Electrophysiological studies have identified numerous cell-autonomous effects of CRF on 
dopamine neurons, including changes in firing rate [27], excitatory currents [10,28,29], and inhibitory currents [8]. CRF also modulates the firing rate of VTA GABA neurons [30] and regulates presynaptic inhibitory and excitatory input onto dopamine neurons [11,31]. These electrophysiological effects are mediated by activation of the CRF-R1 [8,27,31] and CRF-R2 [10,29] or both [11]. Recent evidence illustrates regional differences in how CRF is released within the VTA [22] and that CRF regulates afferent input in a pathway-selective manner [3]. Together, these prior studies indicate that the behavioral effects of CRF in the VTA likely arises from a complex interplay of the neuropeptide's actions on a diverse set of targets within the midbrain.

Exposure to abused substances elicits an array of intrinsic and synaptic changes within the VTA [32]. Prior drug experience increases CRF receptor levels within the VTA [9,33,34], and alters the electrophysiological effects of CRF on VTA dopamine neurons [8,10,11,31]. As such, the cocaine-mediated loss in the capacity for CRF to regulate motivation could be mediated by a drug-induced alteration in which CRF engages a local circuit to reverse its inhibitory influence on reward-evoked dopamine release. Collectively, our data illustrates that both contingent and non-contingent exposure to abused drugs functionally alters the manner by which CRF acts within the midbrain to control dopamine-dependent behavior.

\section{Acknowledgements:}

I.O., M.M.D., and M.J.F. performed the experiments. I.O., M.M.D., M.J.F., and M.J.W. analyzed the data. I.O. and M.J.W. designed the experiments. M.J.W wrote the first draft of the manuscript and I.O., M.M.D, and M.J.F. made revisions. This work was funded by NIH grants to M.J.W. (DA033386 and DA042362).

\section{References:}

1 Sarnyai Z, Shaham Y, Heinrichs SC. The role of corticotropin-releasing factor in drug addiction. Pharmacological reviews. 2001;53(2):209-43.

2 Hollon NG, Burgeno LM, Phillips PE. Stress effects on the neural substrates of motivated behavior. Nat Neurosci. 2015;18(10):1405-12. 
3 Wanat MJ, Bonci A, Phillips PE. CRF acts in the midbrain to attenuate accumbens dopamine release to rewards but not their predictors. Nat Neurosci. 2013;16(4):383-5.

4 Bryce CA, Floresco SB. Perturbations in Effort-Related Decision-Making Driven by Acute Stress and Corticotropin-Releasing Factor. Neuropsychopharmacology. 2016;41(8):2147-59.

5 Mantsch JR, Baker DA, Funk D, Le AD, Shaham Y. Stress-Induced Reinstatement of Drug Seeking: 20 Years of Progress. Neuropsychopharmacology. 2016;41(1):335-56.

6 Wang B, Shaham Y, Zitzman D, Azari S, Wise RA, You ZB. Cocaine experience establishes control of midbrain glutamate and dopamine by corticotropin-releasing factor: a role in stress-induced relapse to drug seeking. J Neurosci. 2005;25(22):5389-96.

7 Blacktop JM, Seubert C, Baker DA, Ferda N, Lee G, Graf EN, et al. Augmented cocaine seeking in response to stress or CRF delivered into the ventral tegmental area following long-access self-administration is mediated by CRF receptor type 1 but not CRF receptor type 2. J Neurosci. 2011;31(31):11396-403.

8 Beckstead MJ, Gantz SC, Ford CP, Stenzel-Poore MP, Phillips PE, Mark GP, et al. CRF enhancement of GIRK channel-mediated transmission in dopamine neurons.

Neuropsychopharmacology. 2009;34(8):1926-35.

9 Grieder TE, Herman MA, Contet C, Tan LA, Vargas-Perez H, Cohen A, et al. VTA CRF neurons mediate the aversive effects of nicotine withdrawal and promote intake escalation. Nat Neurosci. 2014;17(12):1751-8.

10 Hahn J, Hopf FW, Bonci A. Chronic cocaine enhances corticotropin-releasing factordependent potentiation of excitatory transmission in ventral tegmental area dopamine neurons. J Neurosci. 2009;29(20):6535-44.

11 Williams CL, Buchta WC, Riegel AC. CRF-R2 and the heterosynaptic regulation of VTA glutamate during reinstatement of cocaine seeking. J Neurosci. 2014;34(31):10402-14.

12 Oliva I, Wanat MJ. Operant Costs Modulate Dopamine Release to Self-Administered Cocaine. J Neurosci. 2019;39(7):1249-60.

13 Wanat MJ, Kuhnen CM, Phillips PE. Delays conferred by escalating costs modulate dopamine release to rewards but not their predictors. J Neurosci. 2010;30(36):12020-7.

14 Clark JJ, Sandberg SG, Wanat MJ, Gan JO, Horne EA, Hart AS, et al. Chronic microsensors for longitudinal, subsecond dopamine detection in behaving animals. Nature methods. 2010;7(2):126-9.

15 Heien ML, Khan AS, Ariansen JL, Cheer JF, Phillips PE, Wassum KM, et al. Real-time measurement of dopamine fluctuations after cocaine in the brain of behaving rats. Proc Natl Acad Sci U S A. 2005;102(29):10023-8.

16 Goertz RB, Wanat MJ, Gomez JA, Brown ZJ, Phillips PE, Paladini CA. Cocaine increases dopaminergic neuron and motor activity via midbrain alphal adrenergic signaling. Neuropsychopharmacology. 2015;40(5):1151-62.

17 Salamone JD, Correa M. The mysterious motivational functions of mesolimbic dopamine. Neuron. 2012;76(3):470-85.

18 Fischbach-Weiss S, Janak P. Decreases in Cued Reward Seeking After Reward-Paired Inhibition of Mesolimbic Dopamine. Neuroscience. 2019. 
19 Sombers LA, Beyene M, Carelli RM, Wightman RM. Synaptic overflow of dopamine in the nucleus accumbens arises from neuronal activity in the ventral tegmental area. $\mathrm{J}$ Neurosci. 2009;29(6):1735-42.

20 Boyson CO, Holly EN, Shimamoto A, Albrechet-Souza L, Weiner LA, DeBold JF, et al. Social stress and CRF-dopamine interactions in the VTA: role in long-term escalation of cocaine self-administration. J Neurosci. 2014;34(19):6659-67.

21 Han X, DeBold JF, Miczek KA. Prevention and reversal of social stress-escalated cocaine self-administration in mice by intra-VTA CRFR1 antagonism. Psychopharmacology (Berl). 2017;234(18):2813-21.

22 Holly EN, Boyson CO, Montagud-Romero S, Stein DJ, Gobrogge KL, DeBold JF, et al. Episodic Social Stress-Escalated Cocaine Self-Administration: Role of Phasic and Tonic Corticotropin Releasing Factor in the Anterior and Posterior Ventral Tegmental Area. J Neurosci. 2016;36(14):4093-105.

23 Aberman JE, Ward SJ, Salamone JD. Effects of dopamine antagonists and accumbens dopamine depletions on time-constrained progressive-ratio performance. Pharmacology, biochemistry, and behavior. 1998;61(4):341-8.

24 Cagniard B, Balsam PD, Brunner D, Zhuang X. Mice with chronically elevated dopamine exhibit enhanced motivation, but not learning, for a food reward.

Neuropsychopharmacology. 2006;31(7):1362-70.

25 Hamill S, Trevitt JT, Nowend KL, Carlson BB, Salamone JD. Nucleus accumbens dopamine depletions and time-constrained progressive ratio performance: effects of different ratio requirements. Pharmacology, biochemistry, and behavior. 1999;64(1):21-7.

26 Zhang M, Balmadrid C, Kelley AE. Nucleus accumbens opioid, GABaergic, and dopaminergic modulation of palatable food motivation: contrasting effects revealed by a progressive ratio study in the rat. Behavioral neuroscience. 2003;117(2):202-11.

27 Wanat MJ, Hopf FW, Stuber GD, Phillips PE, Bonci A. Corticotropin-releasing factor increases mouse ventral tegmental area dopamine neuron firing through a protein kinase C-dependent enhancement of Ih. J Physiol. 2008;586(8):2157-70.

28 Tovar-Diaz J, Pomrenze MB, Kan R, Pahlavan B, Morikawa H. Cooperative CRF and alpha1 Adrenergic Signaling in the VTA Promotes NMDA Plasticity and Drives Social Stress Enhancement of Cocaine Conditioning. Cell reports. 2018;22(10):2756-66.

29 Ungless MA, Singh V, Crowder TL, Yaka R, Ron D, Bonci A. Corticotropin-releasing factor requires $\mathrm{CRF}$ binding protein to potentiate NMDA receptors via CRF receptor 2 in dopamine neurons. Neuron. 2003;39(3):401-7.

30 Korotkova TM, Brown RE, Sergeeva OA, Ponomarenko AA, Haas HL. Effects of arousal- and feeding-related neuropeptides on dopaminergic and GABAergic neurons in the ventral tegmental area of the rat. Eur J Neurosci. 2006;23(10):2677-85.

31 Harlan BA, Becker HC, Woodward JJ, Riegel AC. Opposing actions of CRF-R1 and CB1 receptors on VTA-GABAergic plasticity following chronic exposure to ethanol. Neuropsychopharmacology. 2018;43(10):2064-74.

32 Francis TC, Gantz SC, Moussawi K, Bonci A. Synaptic and intrinsic plasticity in the ventral tegmental area after chronic cocaine. Current opinion in neurobiology. 2019;54:66-72. 
33 Vranjkovic O, Van Newenhizen EC, Nordness ME, Blacktop JM, Urbanik LA, Mathy JC, et al. Enhanced CRFR1-Dependent Regulation of a Ventral Tegmental Area to Prelimbic Cortex Projection Establishes Susceptibility to Stress-Induced Cocaine Seeking. J Neurosci. 2018;38(50):10657-71.

34 Zhao-Shea R, DeGroot SR, Liu L, Vallaster M, Pang X, Su Q, et al. Increased CRF signalling in a ventral tegmental area-interpeduncular nucleus-medial habenula circuit induces anxiety during nicotine withdrawal. Nature communications. 2015;6:6770. 